\title{
Transient dynamics between displaced fixed points: an alternate nonlinear dynamical framework for olfaction
}

\author{
Christopher L Buckley*, Thomas Nowotny ${ }^{*}$ \\ From Twentieth Annual Computational Neuroscience Meeting: CNS*2011 \\ Stockholm, Sweden. 23-28 July 2011
}

It is now widely accepted that insects encode odor identity in terms of the stimulus specific rate patterning observed in the projection neurons of the antennal lobe (AL). Several studies have explored nonlinear dynamical frameworks in order to gain general insights into the mechanisms underlying the observed dynamical phenomena. The currently most popular framework is the heteroclinic orbit (HO) developed by Rabinovich and collaborators [1-3]. This framework can account for both the dynamics observed in the locust AL [4] and several desirable computational properties of the $\mathrm{AL}$ and the brain in general [2].

Despite its obvious appeal there has been little work on applying the $\mathrm{HO}$ framework to more detailed conductance based models of olfactory dynamics even though it is in principle possible to obtain $\mathrm{HO}$ dynamics from biologically plausible neurons [5]. However, obtaining proper $\mathrm{HO}$ dynamics with conductance based Hodgkin-Huxley neurons in this work required non-standard assumptions for the synapses [5]. Furthermore, recent experimental evidence has indicated another possible concern about the original HO interpretation. The HO framework assumes that the presence of an odor re-parameterises the connectivity of the AL through input to local neurons (LNs). The mathematical image of this change is a bifurcation from a regime with a single stable fixed point (FP) to a regime with a stable HO. The system approaches the stable $\mathrm{HO}$ (or a limit cycle close to it, in the presence of noise) as long as the odor stimulation continues. The $\mathrm{HO}$ then loses stability and the system returns to the resting state FP when the odor stimulus subsides. Recent experiments involving long periods of odor stimulation have revealed

* Correspondence: c.l.buckley@sussex.ac.uk

CCNR, Informatics, University of Sussex, Falmer, BN1 9QJ, UK that the dynamics of the AL appears to settle into a stable fixed point well before the odor subsides [6]. One explanation for this observation is the so called heteroclinic channel $(\mathrm{HC})$ introduced in [2] in which the HO ends in a stable FP.

Here we describe an alternate nonlinear dynamical framework which provides an appealing account of both the origin and function of rate patterning dynamics. We start by analytically reducing a biologically plausible conductance based model of the AL to a quantitatively equivalent rate model and construct conditions such that the rate dynamics are well described by a single globally stable fixed point. We then straightforwardly describe the AL's response to an odor stimulus as rich transient trajectories between this stable baseline state and an odor specific fixed point. We show how this framework can account for a disinhibitory period observed immediately after the stimulus is removed, for the qualitative differences between the dynamics in the presence and the absence of odor and for the invariance of odor identity to both the duration and intensity of an odor stimulus, as reported in [7]. We compare and contrast this framework with the popular nonlinear dynamical framework of 'winnerless competition' which describes AL dynamics in terms of a HO.

Published: 18 July 2011

\footnotetext{
References

1. Rabinovich M, Huerta R, Laurent G: Neuroscience - Transient dynamics for neural processing. Science 2008, 321(5885):48-50.

2. Rabinovich MI, Huerta R, Varona P, Afraimovich VS: Transient cognitive dynamics, metastability, and decision making. PLOS Comp. Biol 2008, 4(5).

3. Varona, Rabinovich M, Selverston A, Arshavsky Y: Winnerless competition between sensory neurons generates chaos: A possible mechanism for molluscan hunting behavior. Chaos 2002, 12(3):672-677.
} 
4. Laurent $G$, Stopfer M, Friedrich R, Rabinovich M, Volkovskii A, Abarbanel H: Odor encoding as an active, dynamical process: Experiments, computation, and theory. Annual Review of Neuroscience 24.

5. Nowotny T, Rabinovich Ml: Dynamical origin of independent spiking and bursting activity in neural microcircuits. Phys. Rev. Lett 2007, 98(12).

6. Mazor O, Laurent $\mathrm{G}$ : Transient dynamics versus fixed points in odor representations by locust antennal lobe projection neurons. Neuron 2005, 48(4):661-673.

7. Stopfer M, Jayaraman V, Laurent G: Intensity versus identity coding in an olfactory system. Neuron 2003, 39(6):991-1004.

doi:10.1186/1471-2202-12-S1-P237

Cite this article as: Buckley and Nowotny: Transient dynamics between displaced fixed points: an alternate nonlinear dynamical framework for olfaction. BMC Neuroscience 2011 12(Suppl 1):P237.

Submit your next manuscript to BioMed Central and take full advantage of:

- Convenient online submission

- Thorough peer review

- No space constraints or color figure charges

- Immediate publication on acceptance

- Inclusion in PubMed, CAS, Scopus and Google Scholar

- Research which is freely available for redistribution

Submit your manuscript at www.biomedcentral.com/submit 